Aus dem Laboratorium des Krankenhauses.

\title{
Beitrag zu anatomisch-pathologischen Veränderungen in gesunder Haut bei Lenigallol- und Eugallol-Wirkung.
}

Von

\author{
Dr. med. W. Kopytowski, \\ ordinierendem Arzte des St. Lazarus-Hospitals in Warschan.
}

(Hiezu Taf. X.)

Es sind über neun Jahre vergangen, seit das Lenigallol bei der Ekzembehandlung von Prof. Kromayer empfohlen wurde. (Einige neue dermatologische Heilmittel, Derivate des Pyrogallols etc. von Kromayer und Vieth. Monatsh. 1898, Bd. XXVII. Heft 11-20 und Münch. med. Woch. Nr. 6, 1901). Indessen fand dieses Mittel in der Therapie nicht die verdiente Anerkennung. Die pathologisch-anatomischen Veränderungen nach Einwirkung dieses Mittels wurden bis jetzt nicht beschrieben, daher seien einige von mir beobachtete Fälle dieser Art angeführt.

Lenigallol ist eine chemische Verbindung, welche aus dem Pyrogallol erhalten wird, in welchem drei Hydroxylgruppen durch drei Essigsäureradikale ersetzt werden. Graphisch stellt sich dieser Vorhang folgendermaßen dar.

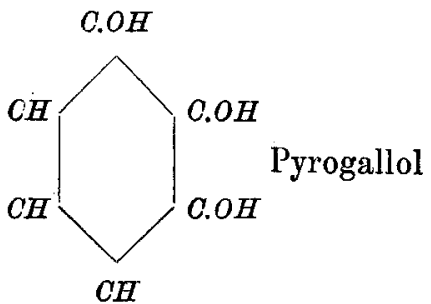

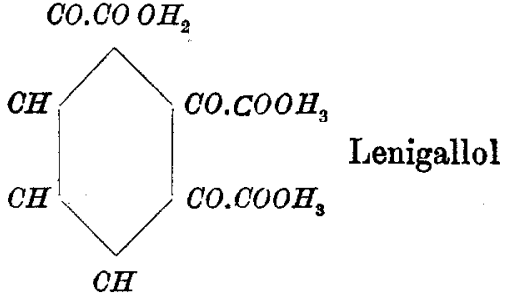


Dieses Mittel stellt ein weißliches, leichtes, in Wasser und Alkohol unlösliches, dagegen in wässerigen Alkalien lösliches Pulver dar. Nach Kromayer soll das Mittel bei Eczem elektiv, nur auf kranke Haut wirken, ohne die gesunde zu betreffen. Das Lenigallol war durch Kromayer mit Zinkpaste $(1: 4)$ empfohlen und soll nur eine Oberflächenwirkung ausüben. Um die Wirkung des Lenigallols zu verstärken und auf die tieferen Schichten zu übertragen, empfahl Kromayer eine Kombination von Lenigallolpaste mit Theer oder Wilkin s on scher Salbe.

So viel mir bekannt, wurden pathologisch-anatomische Veränderungen in der Haut bei Einwirkung des Mittels noch nicht beschrieben. Nach $\mathrm{K}$ romayer soll das Lenigallol reduzierend wirken, wobei sich beim Zusammentreffen desselben mit organischen Säften Pyrogallol bilden soll. Ich hatte Gelegenheit fünf Fälle von Lenigallolwirkung auf gesunde Haut zu untersuchen; sie seien hier in Kürze angeführt und illustrieren teilweise die Wirkungsweise des Mittels. Die Haut stammte stets von jungen Leuten im Alter von 18-34 Jahren. Es wurde eine Vaselinsalbe appliziert von der Konzentration $1: 10$ - 2, 3 bis 4 Tage lang; in der zweiten Serie, in zwei Fällen von der Konzentration $1: 4-3$ und 4 Tage lang. Die Präparate werden in 95\% Alkohol oder in 4\% Formalin und $6 \%$ Sublimat fixiert und in Paraffin eingebettet. Es wurden Serienschnitte von der Dicke $1 / 50-1 / 100 \mathrm{~mm}$ geschnitten und nach den in der Dermatologie üblichen Methoden gefärbt.

Die drei ersten Fällen differierten untereinander sehr wenig, trotzdem eine 1:10 Lenigallolsalbe 2, 3 bis 4 Tage lang appliziert wurde; daher werden sie auch gemeinsam beschrieben.

Die Hornschicht war gewöhnlich vom Präparat abgelöst und in den äußeren Lagen häufig braun-gelb verfärbt. Sie hesteht aus gut verhornten Lamellen und mit sauren Farbstoffen gut tingierten Plättchen ohne Spur von Kernen. An manchen Stellen, in der Nachbarschaft tieferer Hautschichten kamen zuweilen stäbchenförmige, elongierte Kerne vor. Die Öhlsche Schicht tritt nur selten, hie und da in Gestalt eines hellen schmalen Saumes auf. 
Die Körnerschicht stets verdickt, besteht aus $3-\overline{0}$ Reihen Spindelzellen mit reichlichem normalen Keratohyalin. Diese Schicht begrenzte das Präparat in der Regel von oben her (die Hornschicht schilferte sich streifenweise ab).

Die Stachelzellenschicht kaum verändert. Hie und da, meist in den tieferen Lagen traten spärliche Vacuolen auf. Dagegen erschien die Zylinderschicht verdickt und ihr Übergang in die Stachelschicht erfolgte allmählich. Manche hyperplastische Herde des Stratum germinativum zwischen den Papillen bestehen meist aus kleinen kubischen Zellen mit großem Kerne und spärlichem Protoplasma. Keine Mitosen. Dazwischen traten zuweilen schmale, lange, schwach entwickelte, intensiv gefärbte Spindelzellen mit großem Kerne; dieselben wachsen aus der Papillarschicht in die tiefen Lagen des Stratum germinativum hinein. Diese Zellen hatten den Charakter jener Zellen, welche stets in großer Anzahl bei der Einwirkung von Resorcin auf die Haut auftreten; um Wiederholungen zu vermeiden, verweise ich auf meine Arbeit über Resorcinwirkung auf die Haut, wobei ich bemerken muß, daß diese Zellen, meiner Ansicht nach, als in die Epidermis hineinwachsende Gefäßendothelien $\mathrm{zu}$ betrachten sind.

Weiter begegnete ich in dieser Schicht großen Pigmentzellen; unabhängig davon waren die unteren Lagen des Stratum germinativum reich an gelbbraunem, staubförmigem Pigment, welches hauptsächlich in den oberen und unteren Zellabschnitten gruppiert war. Endlich enthielt diese Schicht auch sehr spärliche vereinzelte mehrkernige Leukocyten.

Die Papillarschicht und die oberen Coriumlagen waren leicht ödematös; die Ödeme waren je nach längerer Lenigallolwirkung stärker und manifestierten sich als Erweiterung der zwischen den Bindegewebsfasern gelegenen Lymphräume. Manche Papillen waren wenig infiltriert, die Mehrzahl jedoch fast ohne Infiltrate. Die Gefäße traten in der Mehrzahl der Papillen sehr deutlich auf, ihr Endothel war gequollen. In den Papillen begegnete man öfters zahlreichen Pigmenzellen.

In den oberen Coriumlagen traten stets, meist aus großen einkernigen Leukocyten zusammengesetzte schwache Infiltrate. Die Infiltrate waren in geringem Umfang um die Gefäße loka- 
lisiert. Bei Färbung nach Pappenheim war das grobkörnige Protoplasma mancher Infiltratzellen rot gefärbt. Plasmazellen. Diese Zellen waren denjenigen sehr ähnlich, welche von mir bei der Pyrogallolwirkung beschrieben wurden, und war ihre Zahl viel geringer und sie selbst weniger ausgebildet. Diese Zellen traten deutlich auf nach drei- und viertägiger Lenigallolwirkung. In der Umgebung der Talgdrüsen bemerkte man nach viertägiger Lenigallolwirkung geringe Infiltration.

Die Schweißdrüsen, Hautmuskelfasern, Haare und elastischen Fasern ohne deutliche Veränderungen.

Im vierten und fünften Fall wurde eine starke 1:4 Lenigallolsalbe verwendet. Im vierten Fall wurde die Salbe zweimal in 24 Stunden drei Tage lang gewechselt; im fünften Fall viertägige Wirkung. Diese beiden Fälle boten sehr ähnliche anatomisch-pathologische Veränderungen, werden daher auch zusammen beschrieben.

Die Hornschicht erscheint ungleichmäßig verdickt; ihre äußere Oberfläche ist braungelb gefärbt und vollständig verhornt, d. h. die Hornplättchen sind kernlos. Diese Schicht liegt in Gestalt verschieden dicker Streifen abseits. An anderen Stellen liegt die Hornschicht den tieferen Hautschichten an, ist dann stark verdickt und enthält fast immer stäbchenförmige Kerne. Da die Skrotalhaut nicht glatt und eben ist, sondern zahlreiche kleine Falten wirft, so enthalten diese Vertiefungen stets Auflagerungen parakeratotischer Hornschichten. In der Hornschicht sehr selten solitäre Leukocyten.

Die Öhlsche Schicht tritt hie und $\mathrm{da}$ in Gestalt eines schmalen Streifens auf.

Die Körnerschicht ungleichmäßig entwickelt.

Wo die Hornschicht normal verhornt ist, tritt die Körnerschicht sehr deutlich auf und besteht aus 3 bis 4 Zellreihen mit reichlichem Keratohyalin. Unter den Stellen, wo die zwar verdickte, jedoch abnorm verhornte d. h. kernhaltige Hornschicht sich befindet, ist die Körnerschicht entweder ganz unsichtbar, oder es treten solitäre Zellen mit Keratohyalinspuren auf. 
Die Stachel- und Zylinderschicht ist stark entwickelt. In den unteren Lagen des Stratum germinativum finden sich zahlreiche vacuolenhaltige Zellen. In den unteren Lagen des Stratum spinosum sehen wir an vielen Stellen (an den Schnitten 4 und 5) im Bereiche umgrenzter Partien ( $1 / 2-2$ Papillenbreiten), daß die Zellen der unteren Lagen des Stratum germinativum ihren Zusammenhang verlieren, auseinanderweichen und zerfallen, und dazwischen mehr oder weniger zahlreiche Leukocyten auftreten. Da die unteren Lagen des Stratum germinativum pigmentreich sind, finden wir in diesen Herden auch zahlreiche Pigmentzellen, dagegen sehr wenig seröses Exsudat. Zwischen solchen primären Herden und den älteren, mehr entwickelten, mit partiellem Zerfall von Epidermiszellen, Pigmentzellen und Leukocyten, haben wir eine ganze Reihe Übergangsformen; doch sehen wir stets, daß diese Nester ziemlich klein und spärlich sind, ja, an den Schnitten nach viertägiger Wirkung einer 1:4 Lenigallolsalbe ist der Epidermiszerfall ein beschränkter. Über diesen Herden war die Hornschicht gewöhnlich verdickt, parakeratotisch und bildete mit einigen Reihen Stachelzellen die obere Wand der Herde; die untere Grenze der Zerfallsherde entsprach gewöhnlich den entblößten Papillen, deren Gewebe ödematös aber schwach infiltriert war; dagegegen waren die Infiltrate in dem Corium viel intensiver als in den Papillen.

Die Veränderungen der Papillen und der oberen Coriumlagen waren ganz analog, wie bei schwacher Lenigallolwirkung, aber die entzündlichen Erscheinungen traten viel deutlicher auf: die perivaskulären Leukocyteninfiltrate waren ziemlich groß, ich begegnete mehr nach $\mathrm{P}$ a p p e $\mathrm{n}$ h e im gefärbte Plasmazellen; die Blutgefäße oft bluterfüllt, die Lymphgefäße erweitert, mit feinkörnigen Massen gefüllt usw. Die Umgebung der Haarscheiden, Hautdrüsen, Muskeln öfters infiltriert, die elastischen Fasern obne deutliche Veränderungen. Die Bindegewebszellen färbten sich deutlich und hatten gequollene Kerne. Das ganze Corium am Schnitte mit Leukocyten übersät. Aus obigen Beschreibungen können wir ohne weiteres den Schluß ziehen, daß schwache Lenigallolsalben aut die Haut vorzugsweise keratoplastisch wirken. Meine drei ersten Fälle bringen 
den unumstößlichen Beweis, daß hier zweifellos die günstige Wirkung des Lenigallols auf die Haut in den Vordergrund tritt; es entsteht eine normal verhornte, kernlose Epidermisschicht, deren Dicke die Norm überschreitet. Diese Schicht ist indessen weniger kompakt, d. h. die einzelnen Zellschichten sind untereinander weniger fest gebunden, lösen sich oft in Streifen auf; diese Erscheinung fehlt bei gewöhnlicher Verhornung in normaler Haut, wo die äußeren Schichten als einzelne Zellen oder kleine Zellgruppen abfallen. Auf obige Weise bereits wirkt kein ron mir beschriebenes Mittel (Resorcin, Naphthol $\beta$, Pyrogallol). Es ist sehr wahrscheinlich, daß noch schwächere z. B. $3-5 \%$ Lenigallolsalben, welche ich nicht die Gelegenheit hatte anzuwenden, eine noch günstigere Wirkung ausüben, d. h. Hyperkeratose hervorrufen.

Unabbängig ron seiner Wirkung auf die Epidermis begünstigt das Lenigallol die Entstehung einer dickeren Körnerschicht; dies steht ganz im Einklang mit der Epidermisbildung, da wir wissen, daß die normale Verhornung der Hant von einer normal entwickelten Körnerschicht abhängt. Ferner konnte man eine chromotaktische Wirkung des Lenigallols feststellen, d. h. eine Anziehung des Pigmentes und Wanderung von Pigmentzellen aus den tieferen Hautlagen gegen die Oberfläche der Haut $z u$; doch ist diese Wirkung viel schwächer, als die des Resorcins und selbst des $\beta$-Naphthols.

Die Einwirkung des Lenigallols auf das Getäßendothel und sein Hineinwachsen in das Stratum germinativum ist eine sehr geringe. Die konzentrierteren Salben dagegen, wie $1: 4$ rüfen schon eine intensivere Hautentzündung hervor in Gestalt von Parakeratose der Hornschicht von Ödemen und Zerfall im Stratum germinativum. Die Entzündungserscheinungen sind auch in der Papillarschicht und den oberen Coriumlagen sichtbar, jedoch in viel schwächerem Grade und von relativ geringerer Bedeutung, als bei Pyrogallol. Auch die Bildung von Plasmazellen ist viel geringer als bei Pyrogallol.

Aus den anatomisch-pathologischen Veränderungen ist folglich der Schluß zu ziehen, daß schwache Lenigallolsalben, z. B. $10 \%$ eine eminent keratoplastische, starke dagegen eine schwach keratolytische Wirkung besitzen. 
Zugleich mit dem von Prof. Kromayer bei Ekzem besonders empfohlenen Lenigallol macht Autor in derselben Arbeit (einige neue dermatologische Derivate des Pyrogallols, Chrysarobins, Resorzins von E. Kromayer und U. Vieth Monatshefte für prakt. Dermatologie Bd. XXVII Nr. 1 p. 15) auf ein anderes Pyrogallolderivat, Monoacetat des Pyrogallols aufmerksam :

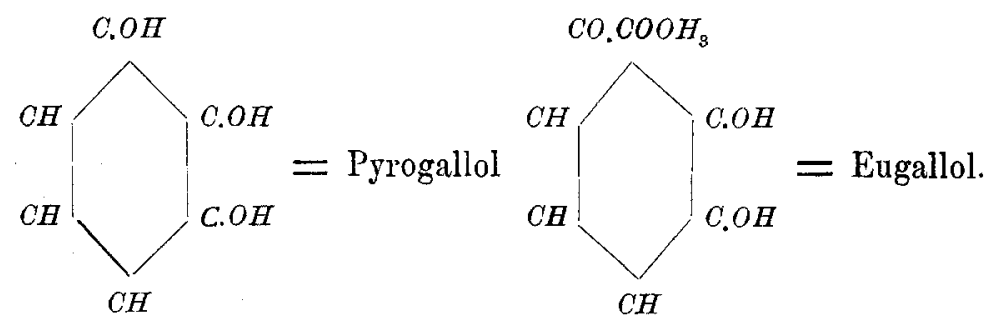

Dieses Mittel wurde gleichfalls in der chemischen Fabrik Knoll \& Co. in Ludwigshafen von Dr. Vieth dargestellt. In seiner Arbeit macht $\mathrm{Kromayer}$ auf die intensive Eugallolwirkung aufmerksam und empfiehlt das Mittel bei Psoriasis wegen seiner günstigen Wirkung selbst auf veraltete Effloreszenzell.

Eugallol bildet eine dicke, dunkle Flüssigkeit, welche im Handel als $33 \%$ Acetonlösung erhältlich ist; das Eugallol ist in Wasser, Alkohol, Chloroform usw. löslich. Mit 50\% Aceton gemischt soll es nach Abdampfen des Acetons einen dünnen, lackartigen Überzug geben.

Die Wirkung des Mittels auf gesunde Haut hatte ich die Gelegenheit in sechs Fällen zu versuchen. Es wurde auch hier die Skrotalhaut junger - im Alter von 19-30 Jahren stehender - Leute mit 1:20 und 1:10 Eugallolsalbe 2, 3, 4 Tage lang behandelt. Die betreffende Hautpartie wurde desinfiziert, mit der Salbe und dann mit hydrophiler Watte bedeckt. Die Salbe wurde täglich gewechselt. Die Präparate waren in Sublimat, Alkohol und Formalin fixiert; die Schnitte $1 / 50-1 / 100 \mathrm{~mm}$ dick; Färbung mittels moderner in der Dermatologie üblicher Methoden.

Um Wiederholungen zu vermeiden, soll die eine Beschreibung die.ersten drei Fälle, wo die 1:20 Salbe 2, 3, 4 Tage lang 
appliziert wurde, umfassen, die andere umfaßt die übrigen drei Fälle von Applikation einer 1:10 Salbe während 2 und 3mal 24 Stunden. Ich mul von vornherein bemerken, daß die Veränderungen der gesunden Haut bei Eugallolwirkung fast immer proportionell der Konzentration und der Wirkungsdauer des Mittels sind, obzwar in einigen Fällen die Veränderungen deutlicher waren bei kürzerer Wirkungsdauer als in anderen bei längerer; dies betrifft hauptsächlich die Wirkung des des Eugallols auf die Hautdrüsen.

Die Wirkung des 1:20 Eugallols in Form einer Vaselinsalbe war folgende:

Die Hornschicht in einzelne, schmale, meist in keinem Zusammenhang mit der Haut stehende Lamellen zerfasert; an zahlreichen Stellen ist sie ganz losgelöst. Hie und da waren einzelne Hornschichtstreifen braun oder braungelb verfärbt; dabei trat die Färbung entweder an den äußeren Hornlamellen oder in der Mitte derselben auf, Die abgeschilferten Hornschichten waren meist kernlos, aber bei längerer Wirkungsdauer des Mittels und Verdickung der Hornschicht traten die Kerne in Gestalt von Stäbchen und Ovalen sehr deutlich auf. Die kernhaltigen Hornschichten lagen öfter den tieferen Hautschichten an als die meist losgelösten kernlosen. Bei längerer Wirkungsdauer des Eugallols traten schon nach dreitägiger Einwirkung Zerfallsherde auf mit Leukocyten, Epithelzellen und Pigmentzellen in verschiedenen Stadien des Zerfalls dieser Elemente.

Die Öhlsche Schicht war unsichtbar; zuweilen konnte man in geringer Ausdehnung ihre Spuren in Gestalt eines durchsichtigen Saumes sehen.

Die Körnerschicht erscheint im Beginn der Wirkung schwachen Eugallols gleichsam leicht verdickt (zuweilen von der Breite von 3-4 Zellreihen), aber schon nach drei Tagen verlieren die Keratohyalinzellen in großer Zahl ihre Kerne, die Zellen atrophieren, wobei anfänglich in deaselben Vacuolen auftreten und das Keratohyalin ungleichmäßig verteilt wird. In den weiteren Stadien bemerkt man nur vereinzelte Keratohyalinzellen, deren Kerne sich des öfteren mit saueren, zuweilen gleichzeitig auch mit basischen Farbstoffen färben. 
Die Stachelschicht ödematös; die tieferen. Zellagen enthalten zahlreiche Vacuolen bereits nach dreitägiger Wirkungsdauer. Zwischen den Zellen zahlreiche vielkernige Leukocyten und selbst verschieden große Zerfallsherde. Diese Herde liegen in den oberen Lagen des Stratum spinosum, gehen jedoch zuweilen in die Hornschicht über, und nehmen so ungleich beide Schichten ein.

Die kubischen Zellen enthalten sehr viel Pigment.

In dieser Schicht bemerkte ich in der ersten Periode der Eugallolwirkung zahlreiche dünne, aus der Papillarschicht hineinwachsende und bis in die tieferen Lagen des Stratum spinosum reichende Spindelzellen. Im weiteren Verlaufe atrophieren diese Zellen; nach viertägiger Wirkung sind kaum ibre Spuren sichtbar.

Nach 3 bis 4 Tagen besteht die Wirkung schwacher Eugallolsalben in Zusammenhangstrennung der Papillarschicht und Epidermis; zugleich weichen die Zellen der tieferen Epidermislagen auseinander, und bilden leere Räume, in welche Leukocyten hineinwandern. In diesen Räumen finden wir außer Leukocyten spärliches seröses Exsudat, dagegen zahlreiche Epithelzellen und Pigmentzellen in verschiedenen Stadien des Zerfalls. In den Anfangsstadien sind diese Herde sehr ähnlich jenen Herden, welche in der Haut infolge lang dauernder Einwirkung starker Lenigallolsalben entstehen. Bei Vergleich entsprechender Präparate konnte ich keinen Unterschied unter ihnen wahrnehmen, doch ist die $1: 20$ Eugallolwirkung viel intensiver, als die einer 1:4 Lenigallolsalbe, da wir der Ablösung der Epidermis von der Bindegewebsschicht der Haut in viel weiterem Umfange begegnen.

Die Papillarschicht und das Corium weisen bereits in den ersten Stadien der Eugallolwirkung einen intensiven Entzündungszustand auf: Gefäßerweiterung, Quellung des Endothels, Erweiterung der Lymphspalten. Plasmazellen treten selten, u. zw. in geringer Anzahl auf, wobei das körnige Protoplasma bei Färbung nach Pappenheim in geringen Quantitäten auftritt, die Zellen selbst wenig entwickelt erscheinen; diesen Zellen begegnete ich erst nach viertägiger Eugallolwirkung. In einem Falle traten in der Papillarschicht und den oberen 
Coriumschichten zablreiche eosinophile Zellen auf, in jedem Gesichtsfeld mehrere.

Die Infiltrate waren nach viertägiger Eugallolwirkung sehr stark, diffus, zumeist im Verlaufe der Gefäße und in der Umgebung von Hautdrüsen. Auch die fixen Bindegewebszellen hatten gequollene Kerne und waren zahlreich.

Die Schweiß- und Talgdrüsen waren äußerst verändert; außer Abschilferung von Epithel und Zerfall von Epithelzellen begegnete ich öfters in den Talgdrüsen reichlichem Exsudat mit angedeutetem Fibrinnetz; in den Schweißdrüsen - außer Zerfall von Epithel, - ausgedehnten Infiltraten mit ein- und mehrkernigen Leukocyten im Drüseninhalt. Erwähnen muß ich, daß man sehr oft in nebeneinander gelegenen Durchschnitten mehrerer Schweißdrüsen eine ungleichmäßige Leukocyteninfiltration beobachten konnte; neben sehr veränderten Schweißdrüsen befanden sich wenig veränderte.

Die Hautmuskelfasern ohne deutliche Veränderungen. Die Glasmembran der Haarscheiden war stets sehr verdickt und zerfasert. Die elastischen Fasern waren im Bereiche der großen Infiltrate gerade gerichtet, verdünnt und in den weiteren Stadien der Entzündung atrophisch.

Bei stärkerer Konzentration des Eugallols (1:10) traten die Entzündungserscheinungen viel früher und stärker auf. Diese Veränderungen waren im Vergleiche mit den durch schwache Salben hervorgerufenen nur quantitativ. Die Hornschicht war gewöhnlich gespalten, undeutlich mit deutlich gefärbten Kernen, am häufigsten fiel sie von den tieferen Hautschichten ab. Keratohyalinzellen fehlen. Zuweilen waren die oberen Schichten des Stratum spinosum gelblich gefärbt; diese Schichten nahmen keine Farbstoffe an, nur zuweilen waren die Kerne schwach tingiert und lagen in einer homogen gelblich gefärbten Masse.

In den oberen Lagen des Stratum spinosum begegnete ich bereits nach zweitägiger Eugallolwirkung zahlreiche kleine Zerfallsherde und Abszesse; in letzteren waren noch stellenweise Scheidewände aus Zellen des Stratum spinosum, welche gedehnt waren und zuweilen an der oberen Wand der Höhle als mehrzellige Fetzen herunterhingen. Die Zellen dieser 
Fetzen waren stark elongiert und in verschiedenen Stadien des Zerfalls begriffen. Bei längerer Einwirkung des Mittels d. $h$. nach 3 und 4 Tagen finden wir schon große, in das Corium tiefgreifende Zerfallsherde. Dadurch wird die Papillarschicht gänzlich zerstört; in den Herden finden wir ein- und mehrkernige Leukocyten, zerfallene Epithelzellen, zerfallene Pigmentzellen und zahlreiche rote Blutkörperchen.

Unter jenen, zu den Hautdrüsen in keinem Verbältnis stebenden Herden, liegen Infiltrate, meist aus großen einkernigen Leukocyten. Diese Infiltrate reichen zuweilen sehr tief in das Corium hinein. Unabhängig von den beschriebenen Herden waren sowohl die ganzen, als die halbierten Schnitte überall mehr oder weniger infiltriert, und zwar mit großen einkernigen Leukocyten.

Außer Infiltraten und Abszessen in der ganzen Haut, in der Stachelschicht und Zylinderschicht, begegnete ich gleichen Veränderungen, wie nach schwachen Eugallolsalben: Ödemen der Zellen, Vacuolen, Wandern des Pigmentes aus der Papillarschicht in die Epidermis und der Leukocyten nach oben usw. Auch hier war der Mangel an Mitosen auffallend.

Ferner waren in der Papillarschicht und den oberen Coriumlagen deutliche Zeichen einer lebhaften Entzündung sichtbar, gleich wie bei schwächeren Engalloldosen. Auch hier Rudimente, nach Papp enheim gefärbte Zellen erkrankter Plasmazellen.

Atrophie der elastischen Fasern in den Infiltraten, Degeneration und Zerfall des Epithels der Hautdrüsen, seröse Exsudate mit Fibrinzusatz in den Talgdrüsen, eiteriges Exsudat in den Schweifdrüsen; Blutaustritte an verschiedenen Stellen des Coriums und der Papillen usw. Alles dies spricht für einen stürmischen Verlauf der durch konzentriertere Eugallolsalbe hervorgerufener Entzündung.

Stellen wir jetzt die Wirkung des Eugallols, Lenigallols und ihres Protoplasts, des Pyrogallols nebeneinander, so sehen wir, daß die Wirkung des Pyrogallols und Eugallols fast gleich ist. Wollte ich auf Grund der spärlichen (6) Fälle, welche ich die Gelegenheit hatte zu untersuchen, eine Differentialdiagnose aufstellen, so würde sich dieselbe auf folgende Punkte reducieren: 
1, tehlt Bildung von Plasmazellen bei Eugallol, dagegen tritt zuweilen Eosinophilie auf; 2, ganz analoge, aber viel intensivere degenerative Veränderungen im Epithel der Hautdrüsen und stärkere Entzündung derselben. Die in Abszessen des Coriums öfter auftretenden Blutaustritte sprechen gleichfalls für stärkere Entzündung bei Engallol als nach Pyrogallol.

Dagegen besitzt das Eugallol, sowie das Pyrogallol sehr wenig Ähnlichkeit mit dem Lenigallol. Seine keratoplastische Wirkung ist nämlich gleich Null, es sei denn ich noch schwächeren Dosen als 5\% Salben; letztere konnten aber nicht erprobt werden. Die eiterbildende (leukotaktische, chromotaktische und erythrotaktische) Wirkung des Engallols tritt auf den ersten Platz hervor, was bei Lenigallol nicht der Fall ist.

Eine Differenzierung zwischen Präparaten nach Einwirkung von Eugallol und Pyrogallol wäre unmöglich, es sei denn beim Auftreten zahlreicher Plasmazellen (Pyrogallol) oder eosinophiler Zellen (Eugallol), was nicht immer stattfindet. Dagegen wäre die Erkennung einer durch Lenigallol gereizten Haut mit stark entwickelter, sei es normaler oder parakeratotischer Epidermis rollkommen möglich.

\section{Erklärung der Abbildungen auf Taf. $X$.}

Fjg. 1. Viertägige Wirkung einer 1:4 Lenigallolsalbe auf gesunde Haut. Zerfall tieferer Schichten des Stratum spinosum und Cylindricum Pigmentreiche Haut. Zeiss Ok. 2, Obj. DD. Hämotoxylin-Eosinfärbung. $A$. Parakeratotische Hornschicht. $B$. Leere Räume im Stratum germinativum. C. Leeres Blutgefäß.

Fig. 2. Viertägige Wirkung einer 1:20 Eugallolsalbe auf gesunde Haut. Hämorrbagische Blasen und Infiltrate in den oberen Coriumlagen. Zeiss. 0k. 3, Hämatoxylin-Eosinfärhung. A. Bläschen. B. Zerfallender Blaseninhalt. $C$. Hämorrhagisches Infiltrat in der Papillarschicht. $D$. Haar. E. Talgdrüse. F. Gefäße.

Fig. 3. Talgdrüse nach dreitägiger Eugallolwirkung 1:10. Zeiss. Ok. 3, Obj. DD. Hämatoxylin-Eosinfärbung. Zerfall des Epithels. $A A$. Seröses Exsudat. $B$. Zerfallende Zelle. $C$. Bindegewebe.

Fig. 4. Schweißdrüse bei dreitägiger Wirkung einer 1:10 Eugallolsalbe. Zeiss. Ok. 3, Obj. DD. Hämatoxylin-Eosinfäroung. Zerfall und Desquamation des Drüsenepithels. A. Infiltrate des Bindegewebes um die SchweiBdrüse. $B B B$. Zerfallende Drüsenzellen. $C$. Infiltrat in einem Drü̊enknäuel. $D$. Spindelzellen des Drüsenbindegewebes. 

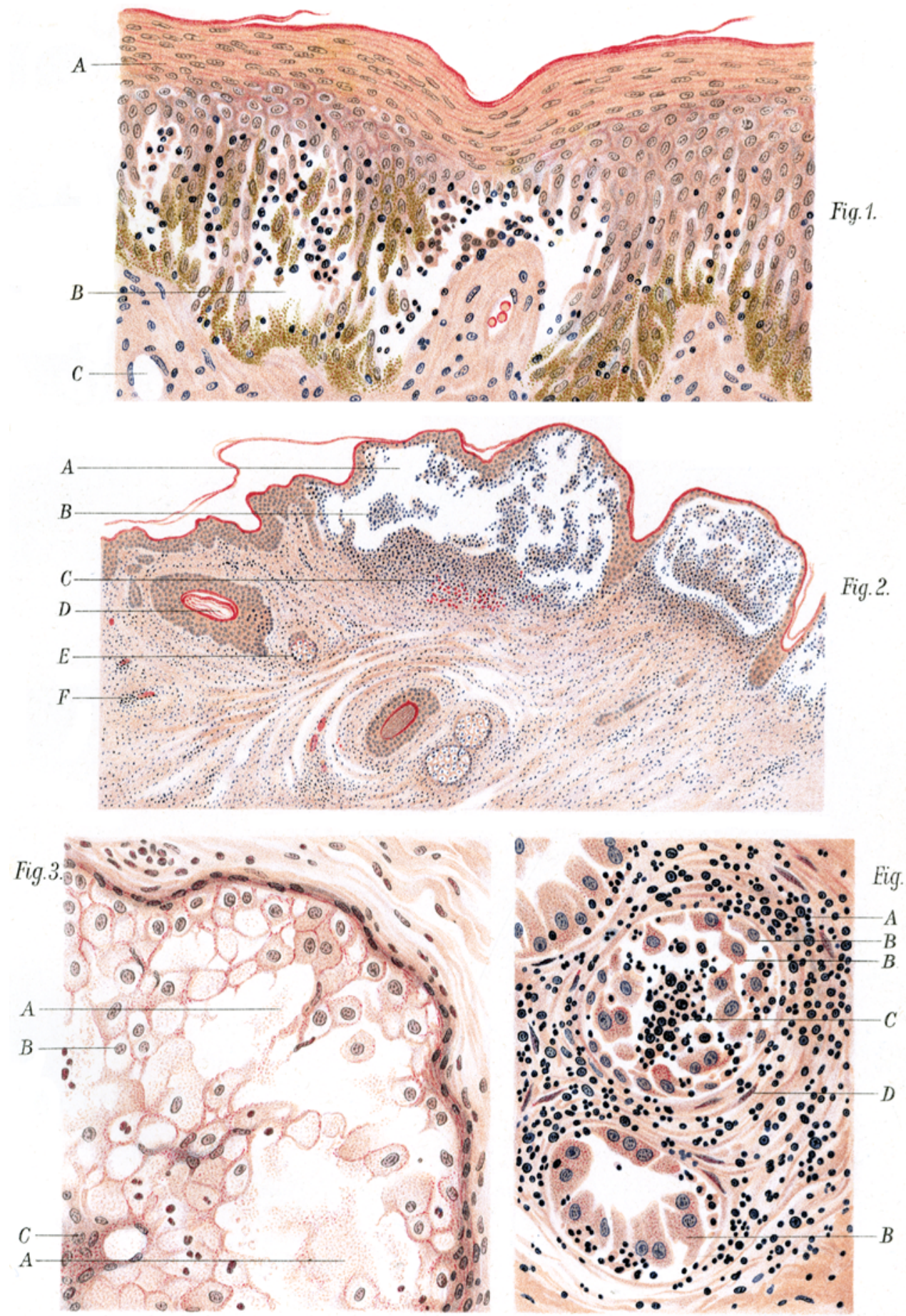